\title{
EFFECTS OF VARIOUS COMPOUNDS ON LIPID PEROXIDATION MEDIATED BY DETERGENT-SOLUBILIZED RAT LIVER NADPH-CYTOCHROME $C$ REDUCTASE
}

\author{
Tetsuya KAMATAKI*, Osamu SUGITA, Shigeru NAMINOHIRA \\ and Haruo KITAGAWA \\ Department of Biochemical Pharmacology, Faculty of Pharmaceutical Sciences, \\ Chiba University, Yayoi-cho, Chiba-shi, Chiba 280, Japan
}

Accepted May 4, 1978

\begin{abstract}
A reconstituted lipid peroxidation system containing NADPH-cytochrome $c$ reductase isolated from detergent-solubilized rat liver microsomes was used to determine the effects of several compounds, including drugs, on the lipid peroxidation activity. EDTA and ferrous ion were essential reyuirements for reconstitution of the activity. The addition of 1,10-phenanthroline to the system containing both EDTA and ferrous ion further enhanced the activily. Pyrocatecol, thymol, $p$-aminophenol, imipramine, $p$-chloromercuribenzoate (PCMB) and $q$-locopherol exhibited strong inhibition, aniline, N-monomethylaniline, aminopyrine, benzphetamine, SKF 525-A and NADP exhibited moderate inhibition, and phenol, benzoic acid, acetanilide and nicotinamide exhibited less or no inhibition at the concentrations lower than $1000 \mu \mathrm{M}$. Metal ions such as $\mathrm{Hg}^{+}, \mathrm{Hg}^{2+}, \mathrm{Co}^{2+}, \mathrm{Cu}^{2+}, \mathrm{Mn}^{2+}$ and $\mathrm{U}^{6+}$ inhibited lipid peroxidation strongly. In addition, $\mathrm{Cd}^{24}, \mathrm{St}^{2+}$ and $\mathrm{Ca}^{2-}$ exhibited less potent to moderate inhibition, and $\mathrm{Ba}^{2+}$ and $\mathrm{Mg}^{2+}$ were without effects on the activity. Among sulfhydryl compounds tested, dithiothreitol inhibited lipid peroxidation to a greater extent than did the other three compounds, glutathione, cysteine and mercaptoethanol.
\end{abstract}

Orrenius et al. (1) first reported that aminopyrine and codeine, which are known to be oxidatively metabolized by a NADPH-dependent enzyme system, inhibited microsomal NADPH-dependent lipid peroxidation. Based on this evidence, they demonstrated the possibility that the inhibition of lipid peroxidation by these drugs was due to competition between the two processes for a common NADPH-oxidizing enzyme. Thereafter, Wills (2) proposed that the inhibition of lipid peroxidation did not appear to be caused by the competition between the two processes since a drug which undergoes hydroxylation reaction by liver microsomes did not necessarily inhibit lipid peroxidation. This idea was further supported by the result that aminopyrine inhibited ascorbate-dependent lipid peroxidation. In addition, non-enzymic lipid peroxidation has also been reported to be inhibited by some drugs (3).

Oshino et al. (4) found that the activity of cyanide sensitive factor (CSF), which catalyzes desaturation reaction of saturated fatty acids, was supported either by NADH or NADPH.

* Present address: Department of Pharmacology, Keio University School of Medicine, Shinanomachi, Shinjuku-ku, Tokyo 160, Japan 
From this and other experimental results, they confirmed that electrons from NADPH were transferred to CSF via NADPH-cytochrome $c$ reductase and cytochrome $b_{5}$. Further, Oshino and Sato (5) proposed the possibility that some compounds including p-cresol, aniline and $p$-aminophenol were oxidatively metabolized by CSF since such were capable of enhancing the reoxidation rate of NADH-reduced cytochrome $\mathbf{b}_{5}$. The latter finding might also suggest that electrons transferred from NADPH to cytochrome $b_{5}$ via NADPHcytochrome $c$ reductase are utilized rapidly when these compounds are added to microsomes and that the enhanced electron flow from NADPH-cytochrome $c$ reductase to cytochrome $b_{5}$ should occur, resulting in inhibition of lipid peroxidation, in a manner similar to that proposed by Orrenius et al (1). In an apparent accordance with this hypothesis, aniline and $p$-aminophenol are reported to be potent inhibitors of microsomal NADPH-dependent lipid peroxidation $(2,6)$. Since many phenol and amine compounds have been established as antioxidants, these compounds were expected to inhibit lipid peroxidation without aids of these possibilities as above described. To confirm the direct effects of these compounds on NADPH-dependent lipid peroxidation, it was necessary to use a purified preparation of an enzyme which is active for NADPH-dependent lipid peroxidation and is free from both cytochrome P-450 and cytochrome $b_{5}$.

Pederson and Aust (7) and Pederson et al. (8) confirmed that the NADPH-dependent lipid peroxidation was catalyzed by NADPH-cytochrome $c$ reductase since the lipid peroxidation activity could be reconstituted using NADPH-cytochrome $c$ reductase isolated from bromelain-treated rat liver microsomes. The proteolytic digestion of microsomal membranes cleaves hydrophobic peptides of the membrane bound enzymes, and the hydrophobic nature of membrane bound enzymes is successfully retained when microsomes are solubilized with detergents (9-12). Therefore, to cstablish the effects of some compounds on the NADPH-cytochrome $c$ reductase mediated lipid peroxidation, we partially purified NADPH-cytochrome $\cdot$ reductase from detergent solubilized microsomes.

Metal ions including $\mathrm{Mn}^{2+}$ are also known to inhibit microsomal NADPH-dependent lipid peroxidation $(13,14)$. Recent studies by Fong et al. (15) have demonstrated that $\mathrm{Mn}^{2+}$ inhibits lipid peroxidation functioning as an efficient superoxide anion scavenger. In this paper, we also report the ability of metal ions to inhibit the NADPH-cytochrome $c$ reductase mediated lipid peroxidation.

\section{MATFRIALS AND METHODS}

Male rats of $\mathrm{Crl}: \mathrm{CD}(\mathrm{SD})$ strain weighing 100 to $150 \mathrm{~g}$ were used throughout this study. In some experiments, the animals were given $0.1 \%$ sodium phenobarbital in the drinking water for three days. All animals were fasted for $48 \mathrm{hr}$ prior to sacrifice. Liver microsomes were prepared as described previously (16). The pooled microsomes were washed once with $1.15 \% \mathrm{KCl}$. The washed microsomes were suspended in redistilled water at a concentration of approx. $30 \mathrm{mg}$ protein per $\mathrm{ml}$ and stored under an atmosphere of nitrogen at $-70^{\circ}$ until usc. Protein was determined by the method of Lowry et al (17). Values represented in the figures and tables are the means of duplicate or triplicate determinations. 


\section{Preparation of substrate lipids}

Lipids used as a substrate of lipid peroxidation were extracted from intact liver microsomes by the method of Folch et al (18). The extracted lipids were dissolved in chloroform at a concentration of about $100 \mathrm{mg}$ lipid phosphorus per $\mathrm{ml}$ and were stored under nitrogen at $-70^{\circ}$. For the assay of lipid peroxidation, the lipid preparation was suspended in redistilled water by sonication three times at 10 second intervals. Lipid phosphorus was determined by the method of Bartlett (19).

Partial purification of $N A D P H$-cytochrome c reductase from rat liver microsomes

The pooled microsomes from phenobarbital-treated rat livers were treated once with 0.1 M pyrophosphate ( $\mathrm{pH}$ 7.4) and were used for purification of NADPH-cytochrome $c$ reductase. The pyrophosphate-extracted microsomes were solubilized with Emulgen 913. NADPH-cytochrome $c$ reductase was purified from the solubilized microsomes by means of DEAE-Sephadex (A-50) column, $\omega$-amino- $n$-octyl Sepharose $4 \mathrm{~B}$ column and hydroxylapatite column as reported previously (20) except that $150 \mathrm{mM}$ rather than $125 \mathrm{mM} \mathrm{KCl}$ was added to the solubilized microsomes and to the buffer for equilibrating DEAE-Sephadex column. Sizes of DEAE-Sephadex, $(0$-amino- $n$-octyl Sepharose 4B and hydroxylapatite columns were $5.0 \times 10.0 \mathrm{~cm}, 1.5 \times 7.5 \mathrm{~cm}$, and $3.0 \times 10.0 \mathrm{~cm}$, respectively. Further, the eluate from the column of $\omega$-amino-n-octyl Sepharose $4 \mathrm{~B}$ was concentrated with polyethyleneglycol $(6,000)$, followed by dialyzation against $20 \mathrm{mM} \mathrm{K}$-phosphate $(\mathrm{pH} 7.25)$ containing $20 \%$ glycerol for $24 \mathrm{hr}$. The specific content of the purified NADPH-cytochrome $c$ reductase was 21.05 unit/mg protein and the recovery of the activity was $24.4 \%$. This NADPH-cytochrome $c$ reductase preparation was free from cytochrome P-450 and cytochrome $b_{5}$. The NADPH-cytochrome $c$ reductase activity was measured by the method of Phillips and Langdon (21). A unit of NADPH-cytochrome $c$ reductase activity is defined as one $/$ mole cytochrome $c$ reduced per min.

Assay of NADPH-cytochrome c reductase mediated lipid peroxidation activity

A typical incubation mixture for the assay of NADPH-cytochrome $c$ reductase mediated lipid peroxidation consisted of $0.25 \mathrm{M}$ Tris- $\mathrm{HCl}$ (pH 6.8), $0.25 \mathrm{M} \mathrm{NaCl}, 0.5 /$ M FMN, $25 / / \mathrm{M}$ EDTA, $50 / / \mathrm{M} \mathrm{Fe}\left(\mathrm{NH}_{4}\right)_{2}\left(\mathrm{SO}_{4}\right)_{2} \cdot 6 \mathrm{H}_{2} \mathrm{O}, 3 \mathrm{mM}$ extracted lipids (as lipid phosphorus) and 0.01 unit of NADPH-cytochrome $c$ reductase. The reaction was started by the addition of $0.2 \mathrm{mM}$ NADPH. The lipid peroxidation activity was estimated by determining malondialdehyde formed by the thiobarbituric acid method as described previously (20). Incubations were carried out in the presence and absence of NADPH-cytochrome $c$ reductase. The valucs for NADPH-cytochrome $c$ reductase mediated lipid peroxidation were thus obtaincd by subtracting the optical density at $532 \mathrm{~nm}$ of the control (--NADPH-cytochrome $c$ reductase) from the experimental incubation ( -1 NADPH-cytochrome $c$ reductase). A mmolar extinction coefficient of $156 \mathrm{mM}^{-1} \mathrm{cn}^{-1}$ (13) for malondialdehyde-thiobarbituric acid complex was used for calculating the lipid peroxidation activity.

\section{Materials}

Emulgen 913 , a non-ionic detergent, was kindly provided by Kao-Atlas Co., Japan, 
and $\alpha$-tocopherol was a gift from Eisai Co., Japan. NADP and NADPH were purchased from Boehringer Mannheim, FMN from Wako Pure Chemicals, Japan. Sepharose 4B and DEAE-Sephadex (A-50) were purchased from Pharmacia Fine Chemicals Co., hydroxylapatite (Bio Gel HT) from Bio-Rad. The $\omega$-amino-n-octyl derivative of Sepharose 4B was prepared from cyanogen bromide-activated Sepharose $4 \mathrm{~B}$ and 1,8-diamino-octane by a method described by Cuatrecasas (22). Commercial aniline was redistilled under vacuum and the distillate was stored under nitrogen at $20^{\circ}$. Other chemical reagents of analytical grade were purchased from commercial sources and were used without further purification.

\section{RESULTS}

The requirements for reconstitution of the NADPH-cytochrome $c$ reductase mediated lipid peroxidation activity were examined (Table 1). As was demonstrated by Pederson and Aust (7) and Pederson et al. (8), EDTA as well as ferrous ion was an essential requirement for the activity. In addition, $\mathrm{NaCl}$ was also required for the maximal activity. FMN exhibited a slight enhancement of the activity. To determine the concentration of EDTA for the maximal activity, the effect of varying concentrations of EDTA on the lipid peroxidation activity was examined in the presence of $50 / / \mathrm{M}$ ferrous ion ( $\mathrm{H} i g .1)$. A $25, " \mathrm{M}$ EDTA concentration was found to produce a maximal enhancement of the activity. In a preceeding paper (20), we reported that 1,10-phenanthroline and 2,2'-bipyridine were also capable of supporting lipid peroxidation aclivity but to extents lesser than EDTA when such were added in place of EDTA. Fig. 2 shows the effects of 1,10-phenanthroline and 2,2'bipyridine on lipid peroxidation activity. In this experiment, either of these chelating reagents was added to the incubation mixture containing EDTA, ferrous ion and other necessary components. As can be seen, 1,10-phenanthroline exhibited the enhancement of lipid peroxidation at a concentration near $25 / / \mathrm{M}$. 2,2'-Bipyridine also enhanced the activity but the enhancement increased graduatly with increase in the concentrations.

Wills (2) reported that phenols and aromatic amines are potent inhibitors of NADPH-

TABLt 1. Requirements for lipid peroxidation activity in the reconstituted system

\begin{tabular}{lc}
\hline Incubation conditions & Lipid peroxidation activity: \\
Complete system" & 1.03 \\
-Fe' & 0.02 \\
-EDTA & 0.00 \\
-FMN & 0.98 \\
- NaCl & 0.56 \\
-EDTA, Fe ${ }^{-+}$ & 0.00 \\
-FMN, NaCl & 0.49 \\
\hline
\end{tabular}

a) umole malondialdehyde innit NADPH-cytochrome c reductase $10 \mathrm{~min}$

b; Complete system for lipid peroxidation activity contained all necessary components described in MATERIALS AND METHODS. 


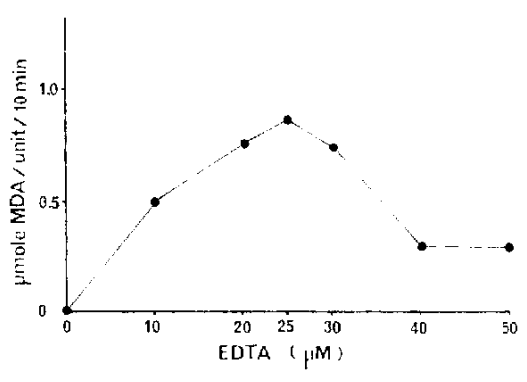

FiG. 1. Enhancement of NADPH-cytochrome $c$ reductase mediated lipid peroxidation by EDTA. The incubation system contained all necessary components as described in MATERIALS AND METHODS except that varying concentrations of EDTA were used. MDA; malondialdehyde.

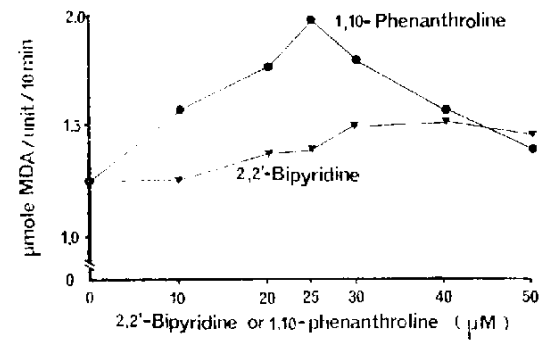

Fici. 2. Effects of 1,10-phenanthroline and 2,2'-bipyridine on NADPH-cytochrome $c$ reductase mediated lipid peroxidation. The incubation system contained all necessary components described in MATERIALS AND METHODS except that various concentrations of 1,10 phenanthroline or 2,2'-bipyridine were added. MDA; malondialdehyde.

dependent lipid peroxidation in rat liver microsomes. The elfects of various compounds on the NADPH-cytochrome $c$ reductase mediated lipid peroxidation are shown in Table 2. Phenol compounds such as pyrocatecol and thymol and some amine compounds such as $p$-aminophenol and imipramine strongly inhibited the NADPH-cytochrome $c$ reductase mediated lipid peroxidation. Other amine compounds, aniline, $\mathrm{N}$-monomethylaniline, aminopyrine, benzphetamine and SKF 525-A also inhibited the activity but to lesser extents than $p$-aminophenol and imipramine. Acetanilide and ethylmorphine did not inhibit lipid peroxidation at concentrations lower than $1000, \ldots \mathrm{M}$. Wills (2) also reported that about $91 \%$ inhibition of microsomal lipid peroxidation was attainable by addition of $1000 \mu \mathrm{M}$ PCMB. Our experiments have shown that $0.1 /$ M PCMB inhibited about $51 \%$ of the NADPH-cytochrome $c$ reductase mediated lipid peroxidation activity, and $10 \mu \mathrm{M}$ PCMB completely inhibited the activity. $\alpha$-Tocopherol is a widely used antioxidant drug. As was expected, $\alpha$-tocopherol at concentrations below $5, \mu \mathrm{M}$ inhibited lipid peroxidation completely. Nicotinamide did not inhibit lipid peroxidation at concentrations below $1000,: \mathrm{M}$. NADP is known to be a competitive inhibitor of NADPH-cytochrome $c$ reductase (23). As shown in Table 2, this compound inhibited lipid peroxidation about $20 \%$ and $29 \%$ at $500 / \mathrm{M}$ and $1000 / \mathrm{M}$ concentrations, respectively.

The effects of metal ions on the NADPH-cytochrome $c$ reductase mediated lipid peroxidation were also examined (Tahle 3 ). Known inhibitors of microsomal lipid peroxidation, such as $\mathrm{Co}^{21}$ and $\mathrm{Mn}^{2+}$ also inhibited the NADPH-cytochrome $c$ reductase mediated lipid peroxidation. Other metal ions such as $\mathrm{Hg}^{+}, \mathrm{Hg}^{2+}, \mathrm{Cd}^{2}, \mathrm{Cu}^{2+}$ and $\mathrm{U}^{6+}$ also inhibited lipid peroxidation.

Previous reports from our own $(24)$ and other laboratories $(2,25)$ indicated that microsomal lipid peroxidation is inhibited by glutathione. Our results (24) further showed that cysteine and dithiothreitol also inhibited lipid peroxidation but to extents lesser than gluta- 
TABLE 2. Effects of some compounds on NADPH-cytochrome $c$ reductase mediated lipid peroxidation

\begin{tabular}{|c|c|c|}
\hline Addition & Concn. $(\mu \mathrm{M})$ & Lipid peroxidation $(\%)$ \\
\hline None (control) & - & 100 \\
\hline Phenol & 1000 & 101.0 \\
\hline Pyrocatecol & 250 & 0.0 \\
\hline \multirow[t]{2}{*}{ Thymol } & 250 & 30.3 \\
\hline & 500 & 1.1 \\
\hline Benzoic acid & 1000 & 96.5 \\
\hline \multirow[t]{2}{*}{ Aniline } & 250 & 94.5 \\
\hline & 1000 & 87.4 \\
\hline \multirow[t]{2}{*}{$p$-Aminophenol } & 50 & 34.6 \\
\hline & 100 & 6.7 \\
\hline \multirow[t]{2}{*}{ N-Monomethylaniline } & 750 & 92.5 \\
\hline & 1000 & 80.3 \\
\hline Acetanilide & 1000 & 103.5 \\
\hline \multirow[t]{2}{*}{ Aminopyrine } & 250 & 91.9 \\
\hline & 1000 & 73.8 \\
\hline \multirow[t]{2}{*}{ Imipramine } & 500 & 21.7 \\
\hline & 1000 & 13.1 \\
\hline Ethylmorphine & 1000 & 101.0 \\
\hline \multirow[t]{2}{*}{ Benzphetamine } & 500 & 71.9 \\
\hline & 1000 & 57.9 \\
\hline \multirow[t]{2}{*}{ SKF 525-A } & 500 & 73.8 \\
\hline & 1000 & 39.3 \\
\hline \multirow[t]{2}{*}{$\mathrm{PCMB}$} & 0.1 & 49.0 \\
\hline & 10 & 0.0 \\
\hline \multirow[t]{2}{*}{$\alpha$-Tocopherol } & 1.0 & 75.3 \\
\hline & 5.0 & 0.0 \\
\hline \multirow[t]{2}{*}{ NADP } & 500 & 79.8 \\
\hline & 1000 & 71.1 \\
\hline Nicotinamide & 1000 & 102.8 \\
\hline
\end{tabular}

Control activity was $1.51 / /$ mole $\mathrm{MDA} /$ unit/10 min.

TABLE 3. Effects of metal ions on NADPH-cytochrome $c$ reductase mediated lipid peroxidation

\begin{tabular}{lcc}
\hline Addition & Conen. $(\mu \mathrm{M})$ & Lipid peroxidation $(\%)$ \\
$\mathrm{None}$ (control) & - & 100 \\
$\mathrm{HgCl}$ & 250 & 28.0 \\
& 750 & 0.0 \\
$\mathrm{HgCl}_{2}$ & 250 & 0.0 \\
$\mathrm{CdCl}$ & 500 & 51.7 \\
& 1000 & 25.8 \\
$\mathrm{BaCl}_{2}$ & 1000 & 101.3 \\
$\mathrm{CoCl}_{2}$ & 250 & 2.8 \\
$\mathrm{CaCl}_{2}$ & 1000 & 88.8 \\
$\mathrm{StSO}_{4}$ & 1000 & 89.9 \\
$\mathrm{CuCl}_{2}$ & 500 & 9.0 \\
$\mathrm{MgCl}_{2}$ & 1000 & 103.9 \\
$\mathrm{MnCl}_{2}$ & 250 & 0.0 \\
$\mathrm{UO}_{2}\left(\mathrm{NO}_{3}\right)_{2}$ & 250 & 30.3 \\
& 500 & 15.2 \\
\hline $\mathrm{COntrol}^{2}$ & &
\end{tabular}

Control activity was $1.51 \%$ mole $\mathrm{MDA} /$ unit $/ 10 \mathrm{~min}$. 


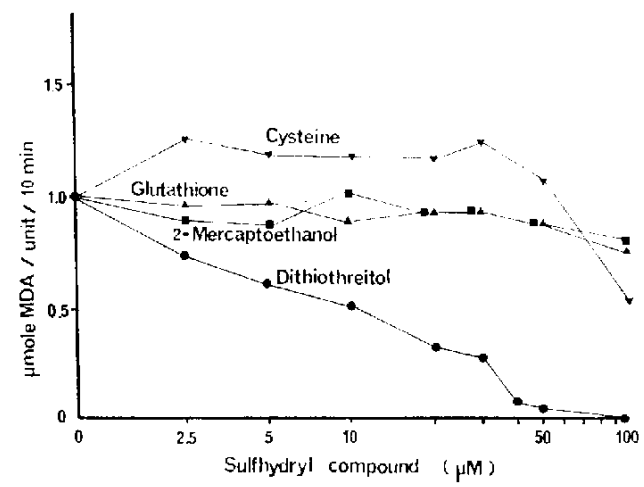

FIG. 3. Inhibition of NADPH-cytochrome $c$ reductase mediated lipid peroxidation by sulfhydryl compounds. MDA; malondialdehyde.

thione. Further, mercaptoethanol did not exhibit significant inhibition. The inhibition of the NADPH-cytochrome $c$ reductase mediated lipid peroxidation by these sulfbydryl compounds is shown in Fig. 3. Our results indicate that dithiothreitol rather than glutathione inhibited lipid peroxidation to a greater extent than did the other sulfhydryl coumpounds.

\section{DISCUSSION}

Effects of drugs, metal jons and sulfhydryl compounds on the NADPH-cytochrome $c$ reductase mediated lipid peroxidation were determined herein. Since the purified preparation of NADPH-cytochrome $c$ reductase did not contain cytochrome P-450 and cytochrome $b_{5}$, the inhibition of lipid peroxidation by these compounds can be free from the effects of these electron transfer enzymes. As was shown in this paper, known inhibitors of microsomal lipid peroxidation also inhibited the NADPH-cytochrome $c$ reductase mediated lipid peroxidation. This finding suggests that inhibition by these compounds is the result of direct effects on lipid peroxidation rather than a competition of the common NADPH-oxidizing enzyme.

It was found that the addition of 1,10-phenanthroline enhanced the NADPH-cytochrome $c$ reductase mediated lipid pcroxidation even in the presence of adequate amounts of EDTA and ferrous ion. This result probably suggests that EDTA and 1,10-phenanthroline enhance lipid peroxidation by different mechanism(s).

Based on the fact that microsomal NADPH-cytochrome $c$ reductase activity was inhibited by some mercurial compounds, it was confirmed that NADPH-cytochrome : reductase contained sulfhydryl groups $(21,23,26)$. In accordance with the previous view, Wills (2) reported that PCMB inhibited microsomal lipid peroxidation and the inhibition of lipid peroxidation was approx. $91 \%$ by $1 \mathrm{mM}$ PCMB. In the present study, we found that the NADPH-cytochrome $c$ reductase mediated lipid peroxidation was inhibited completely by addition of $10 / / \mathrm{M} \mathrm{PCMB}$. The difference between these studies in the ability of PCMB to inhibit lipid peroxidation is probably due to the difference of the total sulfhydryl groups present in these incubation mixtures since we used a small amount of purified NADPHcytochrome $c$ reductase and Wills used microsomes. The organic mercurial PCMB inhibited 
lipid peroxidation activity to a greater extent than did inorganic mercurial compounds, $\mathrm{HgCl}$ and $\mathrm{HgCl}_{2}$. NADPH-cytochrome $c$ reductase, which we purified from detergentsolubilized microsomes, appears to retain a hydrophobic nature. Therefore, the more potent inhibition of lipid peroxidation by PCMB might be caused by the hydrophobic nature of $\mathrm{PCMB}$ as compared to the inorganic mercurials.

Although data are not shown, the inhibitory effects of NADP on lipid peroxidation activity and on NADPH-cytochrome $c$ reductase activity were compared since it was expected that the inhibition by NADP of NADPH-cytochrome $c$ reductase paralleled inhibition of the lipid peroxidation activity. The activity of NADPH-cytochrome $c$ reductase as measured by cytochrome $c$ reduction rate was inhibited by $73 \%$ and $84 \%$ by the presence of $500 \mu \mathrm{M}$ and $1000,4 \mathrm{M}$ NADP, respectively. These results indicate that NADP inhibited cytochrome $c$ reduction rate by $\mathrm{NADPH}$ to a greater extent than NADPH-cytochrome $c$ reductase mediated lipid peroxidation activity. This seems to be rather anomalous and at present unexplainable. However, since a unit of NADPH-cytochrome $c$ reductase $(1000 \mathrm{nmol}$ cytochrome $c$ reduced/min) mediated only one-tenth the capacity in lipid peroxidation $(100$ nmol malondialdehyde formed/unit NADPH-cytochrome $c$ reductase/min), it appears that a rapid flow of electrons to cytochrome $c$ might be one of the causes of the potent inhibition by NADP.

In a previous report from this laboratory (24), we demonstrated that glutathione inhibits microsomal lipid peroxidation to a greater extent than cysteine, dithiothreitol and mercaptoethanol. In this study we observed that dithiothreitol rather than glutathione inhibited the NADPH-cytochrome $c$ reductase mediated lipid peroxidation to a greater extent than the other sulfhydryl compounds. The potent inhibition of microsomal lipid peroxidation by glutathione therefore appeared to be due to the glutathione peroxidase which remained as a contaminant in the washed liver microsomes. In accordance with this assumption, our preliminary experiment indicated that when the microsomes were centrifuged once, a complete removal of glutathione peroxidase activity did not take place. Therefore, the potent inhibition by glutathione seen on liver microsomes seemed to be partly accounted for by the presence of glutathione peroxidase in liver microsomal fraction.

\section{REFERENCES}

1) Orrenius, S., Dallner, G. and Ernster, L.: Inhibition of the TPNH-linked lipid peroxidation of liver microsones by drugs undergoing oxidative demethylation. Biochem. biophys. Res. Commum. 14, 329-334(1964)

2) Wills, E.D.: Lipid peroxide formation in microsomes, Relationship of hydroxylation to lipid peroxide formation. Biochem. J. 113, 333 341 (1969)

3) Archakov, A.I., Panchenko, L.F., Bachmanova, G.I., Chernobrovitna, T.V. and DEVICHENSKI, V.M.: Orientation of electron transfer chains in membranes of the endoplasmic reticulum of liver cells. Dokl. Akal. Nauk USSR 187, 223-226 (1971)

4) Oshino, N., Imai, Y. AND $\mathrm{S}_{\mathrm{ATO}}, \mathrm{R}$.: Function of cytochrome $b_{5}$ in fatty acid desaturation in rat liver microsomes. I. Biochem., Tokyo 69, 155-167 (1971)

5) Oshino, N. AND SATO, R.: Stimulation by phenols of the reoxidation of microsomal bound cytochrome $b_{5}$ and its implication to fatty acid desaturation. J. Biochem., Tokyo 69, $169-180(1971)$ 
6) Gram, T.E. AND Fouts, J.R.: Effect of $\alpha$-tocopherol upon lipid peroxidation and drug metabolism in hepatic microsomes. Arch. Biochem. Biophys. 114, 331-335 (1966)

7) Pederson, T.C. AND Aust, S.D.: NADPH-dependent lipid peroxidation catalized by purified NADPH-cytochrome $c$ reductase from rat liver microsomes. Biochem. biophys. Res. Commun. 48, 789-795 (1972)

8) Pederson, T.C., Butge, J.A. And Aust, S.D.: Microsomal electron transport: The role of reduced nicotinamide adenine dinucleotide phosphate cytochrome $c$ reductase in liver microsomal lipid peroxidation. J. biol. Chem. 248, 7134-7141 (1973)

9) ENomoto, K. AND SATO, R.: Incorporation in vitro of purified cytochrome $b_{5}$ into liver microsomal membranes. Biochem. biophys. Res. Commun. 51, 1-7 (1973)

10) Strittmatter, P., Rogers, M.J. and Spatz, L.: The binding of cytochrome $b_{5}$ to liver microsomes. J. biol. Chem. 247, 7188-7194 (1972)

11) LU, A.Y.H., JUNK, K.W. AND COON, M.J.: Reconstitution of the cytochrome P-450 containing $\omega$-hydroxylation system of liver microsomal three components. $J$. biol. Chem. 244, 3714-3721 (1969)

12) Imai, Y. ANd Minara, K.: Microsomal electron transport system. Protein, Nucleic acid and Enzyme, Tokyo 16, 795-801 (1971)

13) WILls, E.D.: Lipid peroxide formation in microsomes: General considerations. Biochem. J. $113,315-324$ (1969)

14) Kamataki, T. and Kitagawa, H.: Effects of lipid petoxidation on activities of drugmetabolizing enzymes in liver microsomes of rats. Biochem. Pharmacol. 22, 3199-3207 (1.973)

15) Fong, K.L., MCCAY, P.B. AND POYER, J.L.: Evidence for superoxide-dependent reduction of $\mathrm{Fe}^{3+}$ and its role in enzyme-generated hydroxylradical formation. Chem.-Biol. Interns. 15, 77-89 (1976)

16) Kamataki, T. and Kitagawa, H.: Effects of lyophilization and storage of rat liver microsomes on activity of aniline hydroxylase, contents of cytochrome $b_{5}$ and cylochrome P-450 and aniline-induced cytochrome P-450 difference spectrum. Japan. J. Pharmacol. 24, 195-203 (1974)

17) Lowry, O.H., Rosfbrotgh, N.J., Farr, A.L. and Randall. R.J.: Protein measurement with the Folin phenol reagent. J. biol. Chem. 193, 265-273 (1951)

18) Folch, J., Lees, M. and Stanlex, G.H.S.: A simple method for the isolation and purification of total lipids from animal tissues. J. biol. Chem. 226, 497-509 (1956)

19) BArtlett, G.R.: Phosphorous assay in column chromatography. J. biol. Chem. 234, 446-468 (1959)

20) Kamataki, T., Naminohira, S., Sugita, O. and Kitagawa, H.: The lipid peroxidation activity mediated by NADPH-cytochrome $c$ reductase purified from rabbit liver microsomes. Japan. J. Pharmacol. 28, 819-827 (1978)

21) Phillips, A.H. AND LANGdon, R.G.: Hepatic triphosphopyridine nucleotide cytochrome $c$ reductase, isolation, characterization and kinetic studies. J. biol. Chem. 237, 2652-2660 (1962)

22) Cuatrecasas, P.: Protein purification by affinity chromatography. J. biol. Chem. 245, 30593065 (1970)

23) Williams, C.H. AND Kamin, H.: Microsomal triphosphopyridine nucleotide-cytochrome $c$ reductase of liver. J. biol. Chem. 237, 587-595 (1962)

24) Kamatakr, T., Sugita, O., Ozawa, N. and Kitagawa, H.: Stabilization and induction of a lipid peroxidation inhibitor present in the soluble fraction of rat liver homogenate. Toxicol. appl. Pharmacol. 40, 283-290 (1977)

25) Christophersen, B.O.: The inhibitory effecl of reduced glutathione on the lipid peroxidation of the microsomal fraction and mitochondria. Biochem. J.106, $515 \cdots 522$ (1968)

26) Franklis, M.R. And Esiabrouk, R.W.: On the inhibitory action of mersalyl on the microsomal drug hydroxylation. A rigid organization of the electron transport chain. Arch. Biochem. Biophys. 143, 318-329 (1971) 